UDC 614.31: 633.1

DOI: $10.21668 /$ health.risk/2021.3.08.eng

Research article

\title{
DEOXINIVALENOL AS A RISK FACTOR OF FOOD GRAIN CONTAMINATION: MONITORING RESULTS OF 1989-2018 YEARS HARVESTS IN RUSSIAN FEDERATION
}

\author{
I.B. Sedova ${ }^{1}$, L.P. Zakharova ${ }^{1}$, M.G. Kiseleva ${ }^{1}$, Z.A. Chalyy ${ }^{1}$, A.N. Timonin ${ }^{1}$, \\ T.V. Aristarkhova ${ }^{1}$, L.V. Kravchenko ${ }^{1}$, V.A. Tutelyan ${ }^{1,2}$ \\ ${ }^{1}$ Federal Research Centre of Nutrition, Biotechnology and Food Safety, 2/14 Ustinskiy proezd, Moscow, 109240, \\ Russian Federation \\ ${ }^{2}$ I.M. Sechenov First Moscow State Medical University of the Ministry of Health of the Russian Federation, \\ 2 Bldg., 8 Trubetskaya Str., Moscow, 119991, Russian Federation
}

The paper dwells on the results obtained via long-term monitoring over food grain (wheat, barley, corn, oats, and rye) contamination with mycotoxin deoxynivalenol (DON). From 1989 to 2018 6,800 grain samples from Central, Southern, Volga, Ural, Siberian, North-Caucasian, Far Eastern, and Northwestern Federal Districts (FD) of the RF were analyzed. Depending on a year harvest, DON occurrence varied from 0 to $42 \%$ and maximum toxin content reached $6.65 \mathrm{mg} / \mathrm{kg}$. Over the whole examined period $10 \%$ samples turned out to be contaminated and one forth of them contained the toxin in quantities exceeding maximum permissible levels (MPL). DON occurrence amounted to $24-42 \%$ in years of mass epiphytoties (1989, 1992 and 1993) as well as in crops gathered in 2014 and 2017; DON was detected in quantities exceeding MPL in 9-27\% of examined samples in those years. $78 \%$ contaminated samples came from Southern and North-Caucasian FD and another $10 \%$ were from Far Eastern FD. A significant correlation between DON occurrence and a number of rainy and sunny days in May was established on the example of wheat samples from Krasnodar region. Analysis of contamination dynamics has revealed that over the last years there has been an ascending trend in frequency of DON detection in wheat that came not only from regions where Fusarium head blight was widely spread but also from regions in North-western, Siberian and Volga FD. Health risks related to DON intake with wheat grains processing products were assessed; the assessment revealed that DON intake higher than tolerable daily intake (TDI) for the residents of Southern and North-Caucasian FD in 1992, 1993, 2014 and 2017.

Average occurrence of DON was 4.2; 11.9; 3.0 and $0.6 \%$ for barley, corn, rye, and oats samples and its maximum contents amounted to $8.95 ; 0.95 ; 0.96$ and $0.44 \mathrm{mg} / \mathrm{kg}$ accordingly. Just as it was the case with wheat, the most of contaminated samples came from Southern, North-Caucasian and Far Eastern FD. Contamination tended to grow for all the examined grains and it calls for relevant measures aimed at controlling food grains safety

Key words: monitoring, mycotoxins, food grain, wheat, barley, oats, corn, rye, Fusarium head blight, occurrence, deoxynivalenol, health risk assessment, tolerable daily intake, weather, correlation analysis.

(c) Sedova I.B., Zakharova L.P., Kiseleva M.G., Chalyy Z.A., Timonin A.N., Aristarkhova T.V., Kravchenko L.V., Tutelyan V.A., 2021

Irina B. Sedova - Candidate of Biological Sciences, Senior researcher at the Laboratory for Enzymology of Nutrition (e-mail: isedova@ion.ru; tel.: +7 (495) 698-53-65; ORCID: https://orcid.org/0000-0002-6011-4515).

Ludmila P. Zakharova - Candidate of Biological Sciences, Senior researcher at the Laboratory for Enzymology of Nutrition (e-mail: zaharova@ion.ru; tel.: +7 (495) 698-53-65; ORCID: https://orcid.org/0000-0001-7355-5259).

Mariya G. Kiseleva - Candidate of Chemical Sciences, Senior researcher at the Laboratory for Enzymology of Nutrition (e-mail:mg_kiseleva@ion.ru; tel.: +7 (495) 698-53-65; ORCID: https://orcid.org/0000-0003-1057-0886).

Zakhar A. Chalyy - Junior researcher at the Laboratory for Enzymology of Nutrition (e-mail: brew@ion.ru; tel.: +7 (495) 698-53-65; ORCID: https://orcid.org/0000-0002-9371-8163).

Andrey N. Timonin - Candidate of Biological Sciences, Junior researcher at the Immunology Laboratory (e-mail: andrey8407@mail.ru; tel.: +7 (495) 698-53-20; ORCID: https://orcid.org/0000-0001-6087-6918).

Tatyana V. Aristarkhova - Researcher at the Laboratory for Metabolomic and Proteomic Analysis (e-mail: aristarhova_tv@ ion.ru; tel.: +7 (495) 698-53-92; ORCID: https://orcid.org/0000-0001-9496-8626).

Lidiya V. Kravchenko - Candidate of Medical Sciences, Leading researcher at the Laboratory for Enzymology of Nutrition (e-mail: kravchenko@ion.ru; tel.: +7 (495) 698-53-65; ORCID: https://orcid.org/0000-0002-9316-4527).

Victor A. Tutelyan - Academician of the Russian Academy of Sciences, Doctor of Medical Sciences, Professor, Head of the Laboratory for Enzymology of Nutrition, research supervisor (e-mail: tutelyan@ion.ru; tel.: +7 (495) 698-53-46; ORCID: https://orcid.org/0000-0002-4164-8992). 
Grain and the processed products are the traditional diet basis of most people in Russia; therefore, grain quality and safety is the most significant element of food safety in the Russian Federation. Phytopathogens, including toxicogenic micromycetes are an integral part of any agricultural biocenosis. Improper crop rotations, technological breakdowns in grain cultivation and low quality of seed grains together with unfavorable weather make for "field" fungi development, micromycetes from Fusarium genus being among the most widely spread ones. Toxic secondary metabolites produced by microfungi, or mycotoxins, pose a health risk. The greatest health risk is associated with chronic intake of mycotoxins in small amounts with food [1-3].

Deoxynivalenol (DON) is the most widely spread fusariotoxin in the world. It was extracted in 1972 in Japan and the USA and later it was confirmed to be a constant contaminant in grain cultures in most regions all over the world. DON is the most frequently detected in wheat, a bit less frequently in corn, barley, rye, and oats, as well as in their processed grain products [4-12]. Its major producers, causing Fusarium head blight, are fungi from several Fusarium species including $F$. graminearum, F. culmorum, $F$. nivale $[3,6,10,11,13]$. Toxin accumulation depends on toxigenic properties of producing fungi strain, climatic and weather conditions, techniques applied to grow and protect plants, and storage conditions [14-16].

High humidity during and after flowering stage of the crop creates auspicious conditions for Fusarium head blight to appear. DON contents in contaminated grain grow starting from flowering and up to milky-wax ripeness and then they fall dramatically during wax and full ripeness [15, 17]. Moderate climate that is typical for North America, China, and Europe is optimal for Fusairum head blight (FHB) spreading in wheat $[2,6,11,16,18]$. In the Russian Federation grain grown in the North
Caucasian, the Southern, and the Far Eastern Federal Districts (FDs) is the most susceptible to the disease $[2,13,15,19]$.

Acute DON poisoning resulted in vomiting in animals studies (DON is also known as vomitoxin due to this fact). DON was established to cause alimentary toxicosis not only among farm animals [11, 16, 20] but among people as well $[2,20]$. Studies revealed that it bonded to ribosome at molecular level and inhibited protein synthesis thus disrupting proper cell functioning [2]. DON in low doses is able to inhibit immunity. However, exposure to the toxin in its lethal dose can result in leukocytosis, bleeding, diarrhea, and endotoxicosis [21]. The Joint FAO - WHO Expert Committee on Food Additives (JECFA) established provisional maximum tolerable daily intake (PMTDI) and acute reference dose of DON and acetylated metabolites (3-acetyl DON and 15-acetyl DON) for people at 1 and $8 \mu \mathrm{g} / \mathrm{kg}$ of body weight a day accordingly; the determined doses were results of toxicological studies [20]. The prevalence of the toxin and irrefutable evidence that it is truly hazardous for human health justified the introduction of hygienic standards for its contents in food in some countries. Codex Alimentarius Commission established international standards for DON contents in cereal grains (wheat, corn and barley) destined for further processing at the level of 2,000 $\mu \mathrm{g} / \mathrm{kg}$ [22]. In the European Union countries maximum permissible levels (MPL) are regulated according to [23]: they should not exceed $1,750 \mu \mathrm{g} / \mathrm{kg}$ in unprocessed durum wheat, oats, and corn; $1,250 \mu \mathrm{g} / \mathrm{kg}$ in other unprocessed grains; $750 \mu \mathrm{g} / \mathrm{kg}$ in cereals and cereals products intended for direct human consumption. In the RF there are Technical Regulations of the Customs Union (TR CU) No. 021/2011 "On food safety"1, and No. $015 / 2011$ "Safety on grain", according to them DON MPLs in food wheat and barley as

${ }^{1}$ TR TS 021/2011. O bezopasnosti pishchevoi produktsii: tekhnicheskii reglament Tamozhennogo soyuza [TR CU 021/2011. On food safety: The Technical Regulations of the Customs Union. Approved by the Decision by the Customs Union Commission on December 9, 2011 No. 880]. KODEKS: the electronic fund for legal and reference documentation. Available at: http: //www. eurasiancommission.org/ru/db/techreglam/Documents/TR \% 20TS \% 20bezopProd.pdf (March 12, 2021) (in Russian).

${ }^{2}$ TR TS 015/2011. O bezopasnosti zerna: tekhnicheskii reglament Tamozhennogo soyuza [TR CU 015/2011. Safety on grain (last edited on September 15, 2017): The Technical Regulations of the Customs Union. Approved by the Decision by the Customs Union Commission on December 9, 2011 No. 874]. KODEKS: the electronic fund for legal and reference documentation. Available at: https://docs.cntd.ru/document/902320395 (March 12, 2021) (in Russian). 
well as their products are equal to $0.7 \mathrm{mg} / \mathrm{kg}$ and $1.0 \mathrm{mg} / \mathrm{kg}$ respectively.

Long-term mycotoxicological monitoring has been accomplished to examine food grain contamination with mycotoxins, both in areas where Fusarium head blight is widely spread and in other regions where grain is grown. This research work focuses on long-term 30-year monitoring results on DON contamination in food grain (wheat, barley, corn, rye, and oats) harvested in 1989-2018 in order to reveal basic health risks and identify activities for those risks to be reduced.

Materials and methods. Food grain samples $(6,800$ overall) were provided by Rospotrebnadzor regional offices from Central, Southern, Volga, Ural, Siberian, North Caucasian, Far Eastern, and Northwestern Federal Districts of the $\mathrm{RF}^{3}$. Experts examined in total 4,009 wheat samples, 1,293 barley samples, 1,020 rye samples, 278 corn samples and 200 oats samples harvested from 1989 to 2018.

Grain samples were taken from homogenous lots stored at grain reception and grain processing facilities in conformity with GOST (State Standard) R ISO 24333-20114; DON contents in samples were determined with enzyme-linked immunosorbent assay (2009-2012), high performance liquid chromatography with diode-array and ultraviolet detection (HPLC-UV) ${ }^{5}$ (1989-2018) and liquid chromatography with tandem mass spectrometry (HPLC-MS/MS) ${ }^{6}$ (2018).

Data were statistically analyzed in IBM SPSS Statistics 23 (Statistical package for social sciences, USA) and Microsoft Office Excel 2007 (Microsoft Corp., USA). DON contents data in samples were presented in simple mean $(M)$ and median $(M e)$ values and 90-th percentile $(90 \%)$ (contamination levels below minimum detectable concentration for the selected procedures $(0.05 \mathrm{mg} / \mathrm{kg})$ were taken as 0$)$. We accomplished two-factor dispersion and correlation analysis to study correlations between frequency of DON contamination in wheat harvested in 2006-2018 in Krasnodar region and temperature and humidity, duration of sunny weather days and precipitations from May to August. Data were analyzed within the Latin square $3 \times 3$ where lines were numbers of samples and columns were years of harvest, values of dependent variables for a selected wheat class were given in cells with Latin alphabet letters, and frequency of DON contamination was a dependent variable (the significance level was 0.1). Additionally, to establish influence of a parameter on the dependent variable in contamination analysis, we applied Mann Whitney non-parametric test after performing class-interval grouping into two class-intervals for each factor, upper and lower levels, 1 and 2 accordingly. If after dispersion analysis there were more than two deviations in null hypothesis and levels for a fixed factor, we applied Tukey's range test to perform multiple comparisons of simple means with the significance level being 0.05 .

Health risks caused by wheat grain being contaminated with DON were assessed on the basis of comparing total daily DON intake with wheat-based foods and PMTDI DON. Estimated daily DON intake with wheat-based foods was calculated as per the following equation (1):

$$
N_{\text {calc }}=\frac{M \cdot P \cdot 1000}{w},
$$

\footnotetext{
${ }^{3}$ The authors express their deep gratitude to personnel of the Federal Service for Surveillance over Consumer Rights protection and Human Well-being and its Regional Centers for Hygiene and Epidemiology for long-term cooperation, selection and provision of food grain samples to be examined by experts from the Federal Research Center of Nutrition, Biotechnology, and Food Safety.

${ }^{4}$ GOST R ISO 24333-2011. Zerno i produkty ego pererabotki. Otbor prob [GOST R ISO 24333-2011.Cereals and cereal products - Sampling]. KODEKS: the electronic fund for legal and reference documentation. Available at: https://docs.cntd.ru/document/1200092274 (March 11, 2021) (in Russian).

${ }^{5}$ MU 5177-90. Metodicheskie ukazaniya po obnaruzheniyu, identifikatsii i opredeleniyu soderzhaniya dezoksinivalenola (vomitoksina) i zearalenona v zerne i zernoproduktakh [Methodical Guidelines 5177-90. Methodical guidelines on detecting, identifying and determining contents of deoxynivalenol (vomitoxin) and zearalenone in grain and grain products]. Available at: http://www.gostrf.com/normadata/1/4293828/4293828870.pdf (March 13, 2021) (in Russian).

${ }^{6}$ MVI 410/4-2020. Metod mul'tidetektsii mikotoksinov v zerne i pervichnykh produktakh ego pererabotki [Measuring procedures 410/4-2020. A procedure for multidetection of mycotoxins in grains and primary produces of its processing]. Moscow, 2020 (in Russian).
} 
where $N_{\text {calc }}$ is estimated daily DON intake ( $\mu \mathrm{g} / \mathrm{kg}$ of body weight); $M$ is the average DON contents in wheat grains $(\mathrm{mg} / \mathrm{kg}) ; P$ is consumption of processed wheat products ( $\mathrm{kg} / \mathrm{day})$; $w$ is body weight $(\mathrm{kg})$; average body weight of a person is taken as $60 \mathrm{~kg} ; 1,000$ is conversion factor to converse into $\mu \mathrm{g}$.

Results and discussion. Wheat grain contamination harvested in 1989-2018 all over the RF. Frequency of detection and average DON contamination in wheat varied from 0 to $42 \%$ and from 0 to $0.43 \mathrm{mg} / \mathrm{kg}$ accordingly depending on a crop year (Table 1). In total, $10.0 \%$ out of 4,009 grain lots harvested from 1989 to 2018 were contaminated; con- tamination levels varied from 0.05 to $6.65 \mathrm{mg} / \mathrm{kg}$ (Table 1). DON contents were higher than MPL in 101 (2.5\%) grain samples.

$26 \%$ samples from 1989 harvest contained DON and its contents exceeded MPL in $9 \%$. Frequency of DON contamination in 1992 was maximum, $42 \%$ with the exceeding of MPL in $27 \%$ cases. The frequency of DON detection in wheat harvested in 1993 was also high, it reached up to $24 \%$ of the examined samples, and a share of samples with DON contents being higher than MPL reached $15 \%$. DON content in contaminated samples varied from 0.05 to $6.65 \mathrm{mg} / \mathrm{kg}$ in 1998 ; from 0.05 to $5.63 \mathrm{mg} / \mathrm{kg}$ in 1992; and from 0.1 to $3.95 \mathrm{mg} / \mathrm{kg}$ in 1993 .

Table 1

Frequency and levels of DON contamination in wheat harvested in 1989-2018

\begin{tabular}{|c|c|c|c|c|c|c|c|}
\hline \multirow{3}{*}{ Year } & \multicolumn{3}{|c|}{ Number of samples } & \multirow{3}{*}{$\begin{array}{l}\text { DON contents } \\
\text { in contaminated } \\
\text { samples, } \mathrm{mg} / \mathrm{kg}\end{array}$} & \multicolumn{3}{|c|}{ DON content in total samples, $\mathrm{mg} / \mathrm{kg}$} \\
\hline & \multirow{2}{*}{$\begin{array}{l}\text { total, } \\
\text { items }\end{array}$} & $\begin{array}{c}\text { Contaminated } \\
\text { DON }\end{array}$ & $\begin{array}{l}\text { containing DON } \\
\text {-exceeding MPL }\end{array}$ & & \multirow[t]{2}{*}{ M } & \multirow[t]{2}{*}{$M e$} & \multirow[t]{2}{*}{$90 \%$} \\
\hline & & \multicolumn{2}{|c|}{ items (\% of the total number) } & & & & \\
\hline 1989 & 57 & $15(26)$ & $5(9)$ & $0.05-6.65$ & 0.23 & 0 & 0.44 \\
\hline 1990/91 & 67 & $4(6)$ & $1(1)$ & $0.05-0.74$ & 0.02 & 0 & 0 \\
\hline 1992 & 139 & $59(42)$ & $37(27)$ & $0.05-5.63$ & 0.43 & 0 & 1.06 \\
\hline 1993 & 156 & $38(24)$ & $24(15)$ & $0.10-3.95$ & 0.22 & 0 & 0.63 \\
\hline 1994 & 254 & $16(6)$ & $6(2)$ & $0.17-0.96$ & 0.03 & 0 & 0 \\
\hline 1995 & 169 & $11(6)$ & $0(0)$ & $0.07-0.70$ & 0.03 & 0 & 0 \\
\hline 1996 & 120 & $15(13)$ & $0(0)$ & $0.06-0.70$ & 0.02 & 0 & 0.07 \\
\hline 1997 & 137 & $15(11)$ & $1(0.7)$ & $0.05-1.14$ & 0.02 & 0 & 0.05 \\
\hline 1998 & 126 & $12(10)$ & $1(0.8)$ & $0.05-1.09$ & 0.03 & 0 & 0.03 \\
\hline 1999 & 132 & $0(0)$ & $0(0)$ & $<0.05$ & 0 & 0 & 0 \\
\hline 2000 & 222 & 6(3) & $1(0.5)$ & $0.09-0.77$ & 0.01 & 0 & 0 \\
\hline 2001 & 252 & $12(5)$ & $0(0)$ & $0.05-0.62$ & 0.01 & 0 & 0 \\
\hline 2002 & 158 & $6(4)$ & $1(0.6)$ & $0.05-0.78$ & 0.01 & 0 & 0 \\
\hline 2003 & 375 & $5(1)$ & $0(0)$ & $0.05-0.07$ & 0 & 0 & 0 \\
\hline 2004 & 213 & $2(1)$ & $0(0)$ & $0.07 ; 0.08$ & 0 & 0 & 0 \\
\hline 2005 & 147 & $12(8)$ & $0(0)$ & $0.07-0.69$ & 0.02 & 0 & 0 \\
\hline 2006 & 85 & $11(13)$ & $0(0)$ & $0.05-0.34$ & 0.02 & 0 & 0.06 \\
\hline 2007 & 98 & $9(9)$ & $1(1)$ & $0.06-0.91$ & 0.05 & 0 & 0 \\
\hline 2008 & 73 & $4(5)$ & $1(1.4)$ & $0.06-1.03$ & 0.02 & 0 & 0 \\
\hline 2009 & 109 & $8(7)$ & $0(0)$ & $0.06-0.12$ & 0.01 & 0 & 0 \\
\hline 2010 & 122 & $10(8)$ & $2(2)$ & $0.06-1.26$ & 0.03 & 0 & 0 \\
\hline 2011 & 158 & $13(8)$ & 0 & $0.05-0.44$ & 0.01 & 0 & 0 \\
\hline 2012 & 34 & $0(0)$ & 0 & $<0.05$ & 0 & 0 & 0 \\
\hline 2013 & 111 & $9(8)$ & 0 & $0.05-0.52$ & 0.01 & 0 & 0 \\
\hline 2014 & 57 & $21(37)$ & $6(10)$ & $0.07-5.85$ & 0.29 & 0 & 0.66 \\
\hline 2015 & 64 & $6(9)$ & 0 & $0.05-0.33$ & 0.01 & 0 & 0 \\
\hline 2016 & 154 & $29(19)$ & $2(1.3)$ & $0.05-1.43$ & 0.05 & 0 & 0.54 \\
\hline 2017 & 105 & $32(31)$ & $9(9)$ & $0.05-2.46$ & 0.18 & 0 & 0.51 \\
\hline 2018 & 115 & $18(16)$ & $3(2.6)$ & $0.10-1.27$ & 0.06 & 0 & 0.20 \\
\hline TOTAL & 4,009 & $399(10.0)$ & $101(2.5)$ & $0.05-6.65$ & 0.05 & 0 & 0.05 \\
\hline
\end{tabular}

N o t e : DON MPL, mg/kg, is not higher than 0.7 for wheat (TR CU 015/2011 "Safety of grain”). 
Over the next 20 years from 1993 to 2013 frequency of DON contamination in wheat was relatively rare and varied from 0 to $9 \%$. DON contents higher than MPL were detected in very few cases, and maximum DON content in contaminated samples amounted to $1.26 \mathrm{mg} / \mathrm{kg}$. A diagram that showed harvests distributed as per average DON contents revealed a peak at $0.01-0.03 \mathrm{mg} / \mathrm{kg}$.

Starting from 2014 frequency of wheat grain contamination grew to $9-37 \%$ together with increasing numbers of samples with DON contents being higher than MPL. The only exception was the crop of 2015 due to lack of samples contaminated with DON at levels higher than MPL.

Therefore, wheat harvested in 1999, 2003, 2004 and 2012 turned out to be the least contaminated with DON since a share of contaminated samples didn't exceed $1 \%$ and contamination levels were quite low. Frequency of DON contamination varied from 3 to $13 \%$ in wheat harvested in 1990-1991, 1994-1998, 2000-2002, 2005-2011, 2013 and 2015; average DON contents were from 0 to $0.05 \mathrm{mg} / \mathrm{kg}$; and 90 -th percentile, from 0 to $0.07 \mathrm{mg} / \mathrm{kg}$. Contamination with the toxin that was higher than MPL was detected in isolated samples harvested in 1990-1991, 1997, 1998, 2000, 2002, 2007, 2008 and 2010. A peak in DON contamination was detected not only in mass epiphytoties years $(1989,1992$ and 1993) but also in recent years of 2014, 2016, 2017 and 2018. In these years frequency of DON contamination varied from 16 to $37 \%$; average DON contents, from 0.06 to $0.29 \mathrm{mg} / \mathrm{kg}$; 90 -th percentile, from 0.20 to $0.66 \mathrm{mg} / \mathrm{kg}$. Grain samples with DON contents being higher than MPL were the most frequent in wheat harvested in 1989, 1992, 1993, 2014 and 2017 (9-27\% of all the examined samples).

Wheat contamination harvested in 1999-2018 throughout regions. DON contents in wheat were different depending on the region of grain production (Figure 1). Analysis of distribution of contaminated wheat harvested in 1999-2018 showed that $78 \%$ samples (159 out of 205 contaminated samples) were grown in the Southern FD (Republics of Adygei and Kalmykia, Crimea, Astrakhan region,
Volgograd region, and Rostov region) and the North Caucasian FD (the Republics of Ingushetia, North Ossetia - Alania, KabardinoBalkaria, Karachay-Cherkessia, and Stavropol region); all these regions are primary areas in Russia where Fusarium head blight is widely spread in grains. DON contents varied from 0.05 to $5.85 \mathrm{mg} / \mathrm{kg}$ in these samples. $10 \%$ of contaminated samples were received from the Far Eastern FD and $5 \%$ from the Central FD. Frequency of DON contamination was significantly lower in harvests gathered in the Siberian, the Volga, the Northwestern, and the Ural FDs and amounted to $1-2 \%$.

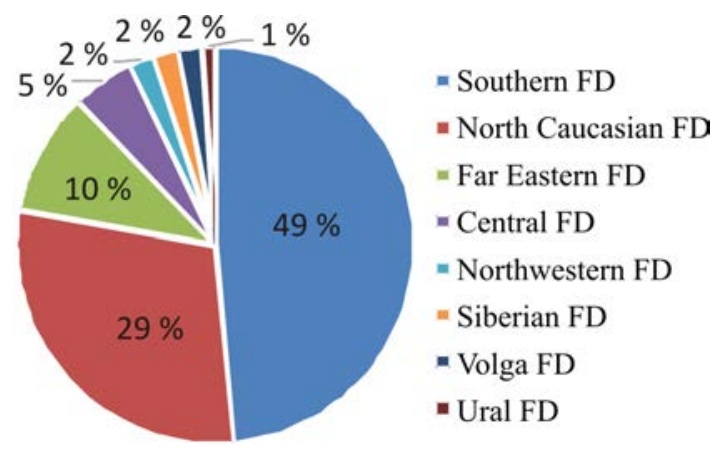

Figure 1. Region distribution of DON contaminated wheat harvested in 1999-2018

( $\%$ of the total contaminated samples)

According to Rosstat data, in 2018 the greatest contributions in total grain harvest belonged to Rostov region, $12.9 \%$; Krasnodar region, $12.4 \%$; and Stavropol region, $9.9 \%$. They were followed by Altai region and Volgograd region, $4.2 \%$ each; Voronezh, Kursk, and Omsk region, from 3.1 to $3.8 \%$; Tatarstan, Lipetsk, Novosibirsk, Tambov, and Orel regionsб from 2 to $2.7 \%$; Orenburg, Penza, Kurgan, Tula, Chelyabinsk, Samara, and Krasnoyarsk regions - from 1.5 to $1.8 \%{ }^{6}$. As said above, grain produced in leading regions in wheat harvesting, namely Rostov, Krasnodar, and Stavropol regions, tended to be more heavily contaminated with DON than grain produced in other regions. Harvested wheat is further processed and consumed not only by people living in the Southern and the North Caucasian FDs but also by people living in other FDs; hence, DON intake due to consumption of contaminated wheat-based products can contribute significantly to DON in- 
take with food products estimated for the country population as a whole.

Dynamics of grain contamination with DON was analyzed on the example of several wheat-producing regions in the RF; Table 2 contains the results.

From 1999 to 2018 wheat samples grown in the Southern FD mostly received from Krasnodar, Rostov, and Volgograd regions, and Adygei and Kalmyk Republics. DON grain contamination in these regions was significantly different depending on the area where grain was grown. For example, grain received from Kalmyk Republic and Volgograd region practically was not contaminated with DON or its content was very low. On the contrary, frequency of the toxin detection was the highest in grain received from Krasnodar region and Adygei Republic. Rostov region was somewhere in between as per this parameter within the Southern FD (Table 2).

Totally, 262 grain samples from Krasnodar region were analyzed; samples contaminated with DON were detected in 10 out of 17 harvests gathered in different years and frequency of detection varied from 0 to $79 \%$ depending on a crop year. In some years a share of samples contained the toxin in concentration higher than MPL varied from $5.9 \%$ (2016) to $42.9 \%$ (2017) and maximum contamination levels reached 3.21 and $5.85 \mathrm{mg} / \mathrm{kg}$.

65 grain samples from Adygei Republic were analyzed and DON was detected in grains from 8 out of 13 harvests gathered in different years in quantities varying from 0.08 to $0.78 \mathrm{mg} / \mathrm{kg}$.

Table 2

Dynamics of wheat DON contamination harvested in 1999-2018 from different FDs in the RF

\begin{tabular}{|c|c|c|c|c|c|c|c|c|c|c|c|c|c|c|c|c|c|c|c|c|}
\hline Region & 1999 & 2000 & 2001 & 2002 & 2003 & 2004 & 2005 & 2006 & 2007 & 2008 & 2009 & 2010 & 2011 & 2012 & 2013 & 2014 & 2015 & 2016 & 2017 & 2018 \\
\hline \multicolumn{21}{|c|}{ The Southern Federal District (694 samples) } \\
\hline $\begin{array}{l}\text { Krasnodar region } \\
\left(n^{* *}=262\right)\end{array}$ & $\begin{array}{c}3 / 0 / 0^{*} \\
(0)\end{array}$ & \begin{tabular}{|c|}
$4 / 0 / 0$ \\
$(0)$
\end{tabular} & $\begin{array}{c}10 / 2 / 0 \\
(20)\end{array}$ & $\begin{array}{c}1 / 0 / 0 \\
(0)\end{array}$ & $\begin{array}{c}5 / 0 / 0 \\
(0)\end{array}$ & $\begin{array}{c}3 / 0 / 0 \\
(0)\end{array}$ & $\begin{array}{c}1 / 0 / 0 \\
(0)\end{array}$ & $\begin{array}{c}15 / 7 / 0 \\
(47) \\
\end{array}$ & $\begin{array}{c}15 / 2 / 0 \\
(13)\end{array}$ & - & - & \begin{tabular}{|c|}
$77 / 7 / 0$ \\
$(9)$
\end{tabular} & $\begin{array}{c}8 / 0 / 0 \\
(0)\end{array}$ & - & $\begin{array}{c}51 / 2 / 0 \\
(4)\end{array}$ & \begin{tabular}{|c|}
$14 / 8 / 2$ \\
$(57)$ \\
\end{tabular} & $\begin{array}{l}9 / 2 / 0 \\
(22) \\
\end{array}$ & $\begin{array}{c}17 / 11 / 1 \\
(65)\end{array}$ & \begin{tabular}{|c|}
$14 / 11 / 6$ \\
$(79)$
\end{tabular} & $\begin{array}{c}15 / 8 / 2 \\
(53)\end{array}$ \\
\hline \begin{tabular}{|l}
$\begin{array}{l}\text { Adygei Republic } \\
(\mathrm{n}=65)\end{array}$ \\
\end{tabular} & - & \begin{tabular}{|c|}
$12 / 2 / 0$ \\
$(17)$ \\
\end{tabular} & $\begin{array}{c}/ 1 / 0 \\
(13)\end{array}$ & \begin{tabular}{|l|}
$5 / 2 / 1$ \\
$(40)$ \\
\end{tabular} & $\begin{array}{c}14 / 1 / 0 \\
(7) \\
\end{array}$ & - & - & - & - & \begin{tabular}{|c|}
$1 / 0 / 0$ \\
$(0)$
\end{tabular} & - & - & $\begin{array}{l}5 / 2 / 0 \\
(40) \\
\end{array}$ & - & \begin{tabular}{|c|}
$1 / 0 / 0$ \\
$(0)$
\end{tabular} & \begin{tabular}{|l|}
$\mathbf{1} / 1 / \mathbf{0}$ \\
$(\mathbf{1 0 0})$ \\
\end{tabular} & \begin{tabular}{|l|}
$1 / 1 / 0$ \\
$(100)$
\end{tabular} & $\begin{array}{c}1 / 0 / 0 \\
(0)\end{array}$ & $\begin{array}{c}1 / 0 / 0 \\
(0)\end{array}$ & \begin{tabular}{|l|}
$1 / 1 / 0$ \\
$(100)$
\end{tabular} \\
\hline \begin{tabular}{|l|}
$\begin{array}{l}\text { Rostov region } \\
(n=271)\end{array}$ \\
\end{tabular} & $\begin{array}{c}25 / 0 / 0 \\
(0) \\
\end{array}$ & \begin{tabular}{|c|}
$8 / 2 / 1$ \\
$(25)$ \\
\end{tabular} & $\begin{array}{c}21 / 8 / 0 \\
(38) \\
\end{array}$ & $\begin{array}{l}8 / 2 / 0 \\
(25) \\
\end{array}$ & $\begin{array}{c}11 / 0 / 0 \\
(0)\end{array}$ & \begin{tabular}{|c|}
$14 / 1 / 0$ \\
$(7)$
\end{tabular} & $\begin{array}{c}25 / 5 / 0 \\
(20) \\
\end{array}$ & $\begin{array}{c}12 / 1 / 0 \\
(8)\end{array}$ & \begin{tabular}{|c|}
$7 / 0 / 0$ \\
$(0)$ \\
\end{tabular} & \begin{tabular}{|c|}
$5 / 0 / 0$ \\
$(0)$
\end{tabular} & \begin{tabular}{|c|}
$34 / 1 / 0$ \\
$(3)$
\end{tabular} & \begin{tabular}{|c|}
$4 / 0 / 0$ \\
$(0)$
\end{tabular} & - & - & $\begin{array}{c}25 / 0 / 0 \\
(0)\end{array}$ & \begin{tabular}{|c|}
$8 / 0 / 0$ \\
$(0)$
\end{tabular} & $\begin{array}{c}28 / 2 / 0 \\
(7)\end{array}$ & \begin{tabular}{|c|}
$21 / 6 / 0$ \\
$(29)$ \\
\end{tabular} & $\begin{array}{c}7 / 0 / 0 \\
(0) \\
\end{array}$ & \begin{tabular}{|c|}
$8 / 0 / 0$ \\
$(0)$
\end{tabular} \\
\hline \multicolumn{21}{|c|}{ The North Caucasian Federal District (333 samples) } \\
\hline \begin{tabular}{|l|}
$\begin{array}{l}\text { Stavropol region } \\
(n=215)\end{array}$ \\
\end{tabular} & \begin{tabular}{|c|}
$3 / 0 / 0$ \\
$(0)$
\end{tabular} & \begin{tabular}{|c|}
$4 / 1 / 10$ \\
$(0)$
\end{tabular} & \begin{tabular}{|c|}
$5 / 0 / 0$ \\
$(0)$ \\
\end{tabular} & \begin{tabular}{|c|}
$2 / 1 / 0$ \\
$(0)$
\end{tabular} & \begin{tabular}{|c|}
$6 / 0 / 0$ \\
$(0)$
\end{tabular} & \begin{tabular}{|c|}
$6 / 0 / 0$ \\
$(0)$
\end{tabular} & \begin{tabular}{|c|}
$4 / 2 / 0$ \\
$(50)$ \\
\end{tabular} & \begin{tabular}{|c|}
$2 / 1 / 10$ \\
$(50)$ \\
\end{tabular} & \begin{tabular}{|c|}
$8 / 0 / 0$ \\
$(0)$ \\
\end{tabular} & \begin{tabular}{|c|}
$1 / 0 / 0$ \\
$(0)$
\end{tabular} & \begin{tabular}{|c|}
$16 / 1 / 0$ \\
$(6)$
\end{tabular} & \begin{tabular}{|c|} 
9/1/0 \\
$(11)$ \\
\end{tabular} & $\begin{array}{c}17 / 2 / 0 \\
(12) \\
\end{array}$ & \begin{tabular}{|c|}
$24 / 0 / 0$ \\
$(0)$
\end{tabular} & \begin{tabular}{|c|}
$19 / 0 / 0$ \\
$(0)$
\end{tabular} & \begin{tabular}{|c|}
$17 / 7 / 3$ \\
$(41)$ \\
\end{tabular} & \begin{tabular}{|c}
$20 / 0 / 0$ \\
$(0)$ \\
\end{tabular} & $\begin{array}{c}19 / 3 / 0 \\
(0)\end{array}$ & \begin{tabular}{|c|}
$15 / 9 / 1$ \\
$(60)$
\end{tabular} & \begin{tabular}{|c|}
$18 / 2 / 1$ \\
$(11)$
\end{tabular} \\
\hline \begin{tabular}{|l} 
Kabardino- \\
Balkaria, North \\
Ossetia - Alania \\
$(n=56)$ \\
\end{tabular} & - & - & - & - & - & - & - & - & $\begin{array}{l}\mathbf{1} / \mathbf{1} / \mathbf{1} \\
(100)\end{array}$ & - & $\begin{array}{c}10 / 4 / 0 \\
(40)\end{array}$ & - & $\begin{array}{c}16 / 6 / 0 \\
(38)\end{array}$ & - & $\begin{array}{l}5 / 2 / 0 \\
(40)\end{array}$ & \begin{tabular}{|c|}
$5 / 4 / 1$ \\
$(80)$
\end{tabular} & $\begin{array}{l}4 / 1 / 0 \\
(25)\end{array}$ & $\begin{array}{c}1 / 0 / 0 \\
(0)\end{array}$ & $\begin{array}{c}2 / 1 / 0 \\
(50)\end{array}$ & $\begin{array}{c}3 / 0 / 0 \\
(0)\end{array}$ \\
\hline $\begin{array}{l}\text { Dagestan, Chech- } \\
\text { nya }(n=44)\end{array}$ & - & \begin{tabular}{|c|}
$2 / 0 / 0$ \\
$(0)$ \\
\end{tabular} & - & - & - & - & \begin{tabular}{|c|}
$11 / 2 / 0$ \\
$(18)$ \\
\end{tabular} & - & $\begin{array}{c}24 / 2 / 0 \\
(8)\end{array}$ & \begin{tabular}{|c|}
$2 / 0 / 0$ \\
$(0)$
\end{tabular} & - & - & $\begin{array}{c}2 / 0 / 0 \\
(0)\end{array}$ & - & - & \begin{tabular}{|c}
$5 / 0 / 0$ \\
$(0)$
\end{tabular} & - & - & - & - \\
\hline \multicolumn{21}{|c|}{ The Far Eastern Federal District (56 samples) } \\
\hline $\begin{array}{l}\text { Amur region } \\
(n=12)\end{array}$ & - & \begin{tabular}{|c|}
$1 / 0 / 0$ \\
$(0)$
\end{tabular} & - & - & - & - & - & - & - & \begin{tabular}{|c|}
$1 / 0 / 0$ \\
$(0)$
\end{tabular} & - & - & - & - & - & \begin{tabular}{|l|}
$\mathbf{1 / 1 / 1 0}$ \\
$(100)$ \\
\end{tabular} & \begin{tabular}{|c|}
$1 / 0 / 0$ \\
$(0)$
\end{tabular} & \begin{tabular}{|l|}
$2 / 2 / 1$ \\
$(100)$
\end{tabular} & \begin{tabular}{|l|}
$3 / 3 / 1$ \\
$(100)$ \\
\end{tabular} & \begin{tabular}{|l|}
$3 / 2 / 0$ \\
$(67)$
\end{tabular} \\
\hline $\begin{array}{l}\text { Primorye } \\
(n=27)\end{array}$ & - & $\begin{array}{c}1 / 0 / 0 \\
(0)\end{array}$ & - & \begin{tabular}{|c|}
$1 / 0 / 0$ \\
$(0)$
\end{tabular} & - & - & \begin{tabular}{|c|}
$4 / 3 / 0$ \\
$(75)$ \\
\end{tabular} & $\begin{array}{l}\mathbf{1 / 1 / 0} \\
(100)\end{array}$ & $\begin{array}{l}\mathbf{1} / \mathbf{1} / \mathbf{0} \\
(100)\end{array}$ & \begin{tabular}{|l|}
$3 / 3 / 1$ \\
$(100)$
\end{tabular} & \begin{tabular}{|c|}
$6 / 2 / 0$ \\
$(33)$
\end{tabular} & \begin{tabular}{|l|}
$\mathbf{1} / \mathbf{1} / \mathbf{1}$ \\
$(\mathbf{1 0 0})$
\end{tabular} & - & - & - & - & - & - & \begin{tabular}{|c|}
$2 / 1 / 1$ \\
$(50)$
\end{tabular} & - \\
\hline \multicolumn{21}{|c|}{ The Central Federal District (693 samples) } \\
\hline $\begin{array}{l}\text { Orel region }(n= \\
150)\end{array}$ & $\begin{array}{c}1 / 0 / 0 \\
(0)\end{array}$ & \begin{tabular}{|c|}
$10 / 0 / 0$ \\
$(0)$
\end{tabular} & - & \begin{tabular}{|c|}
$53 / 0 / 0$ \\
$(0)$
\end{tabular} & $\begin{array}{c}22 / 0 / 0 \\
(0)\end{array}$ & - & \begin{tabular}{|c|}
$3 / 0 / 0$ \\
$(0)$
\end{tabular} & - & - & - & - & $\begin{array}{c}1 / 0 / 0 \\
(0)\end{array}$ & $\begin{array}{c}20 / 1 / 0 \\
(5)\end{array}$ & - & - & - & - & $\begin{array}{l}6 / 1 / 0 \\
(17)\end{array}$ & $\begin{array}{l}5 / 2 / 0 \\
(40)\end{array}$ & \begin{tabular}{|c}
$3 / 0 / 0$ \\
$(0)$
\end{tabular} \\
\hline \begin{tabular}{|l} 
Voronezh region \\
$(n=109)$
\end{tabular} & $\begin{array}{c}7 / 0 / 0 \\
(0)\end{array}$ & \begin{tabular}{|c|}
$10 / 0 / 0$ \\
$(0)$
\end{tabular} & - & \begin{tabular}{|c|}
$7 / 0 / 0$ \\
$(0)$
\end{tabular} & $\begin{array}{c}11 / 0 / 0 \\
(0)\end{array}$ & \begin{tabular}{|c}
$14 / 0 / 0$ \\
$(0)$
\end{tabular} & \begin{tabular}{|c|}
$15 / 0 / 0$ \\
$(0)$
\end{tabular} & \begin{tabular}{|c|}
$5 / 0 / 0$ \\
$(0)$ \\
\end{tabular} & $\begin{array}{c}19 / 3 / 0 \\
(16)\end{array}$ & - & - & \begin{tabular}{|c|}
$1 / 0 / 0$ \\
$(0)$
\end{tabular} & - & - & - & - & - & - & $\begin{array}{c}3 / 0 / 0 \\
(0)\end{array}$ & \begin{tabular}{|c}
$3 / 0 / 0$ \\
$(0)$
\end{tabular} \\
\hline $\begin{array}{l}\text { Other regions } \\
(n=434)\end{array}$ & $\begin{array}{c}42 / 0 / 0 \\
(0)\end{array}$ & $\begin{array}{c}55 / 0 / 0 \\
(0) \\
\end{array}$ & $\begin{array}{c}1 / 0 / 0 \\
(0)\end{array}$ & $\begin{array}{c}15 / 0 / 0 \\
(0) \\
\end{array}$ & $\begin{array}{c}76 / 0 / 0 \\
(0) \\
\end{array}$ & $\begin{array}{c}47 / 0 / 0 \\
(0)\end{array}$ & $\begin{array}{c}27 / 0 / 0 \\
(0) \\
\end{array}$ & $\begin{array}{c}18 / 0 / 0 \\
(0)\end{array}$ & $\begin{array}{c}31 / 0 / 0 \\
(0)\end{array}$ & $\begin{array}{c}28 / 1 / 0 \\
(4)\end{array}$ & $\begin{array}{c}13 / 0 / 0 \\
(0)\end{array}$ & $\begin{array}{c}1 / 0 / 0 \\
(0)\end{array}$ & $\begin{array}{c}17 / 1 / 0 \\
(6)\end{array}$ & - & - & $\begin{array}{c}1 / 0 / 0 \\
(0)\end{array}$ & - & $\begin{array}{c}17 / 1 / 0 \\
(5)\end{array}$ & $\begin{array}{c}19 / 0 / 0 \\
(0)\end{array}$ & $\begin{array}{c}17 / 0 / 0 \\
(0)\end{array}$ \\
\hline $\begin{array}{l}\text { The Volga FD } \\
\text { (331 samples) }\end{array}$ & \begin{tabular}{|c}
$1 / 0 / 0$ \\
$(0)$
\end{tabular} & $\begin{array}{c}29 / 0 / 0 \\
(0)\end{array}$ & $\begin{array}{c}4 / 0 / 0 \\
(0)\end{array}$ & $\begin{array}{c}69 / 0 / 0 \\
(0)\end{array}$ & $\begin{array}{c}23 / 0 / 0 \\
(0)\end{array}$ & $\begin{array}{c}65 / 0 / 0 \\
(0)\end{array}$ & \begin{tabular}{|c|}
$5 / 0 / 0$ \\
$(0)$
\end{tabular} & $\begin{array}{c}5 / 0 / 0 \\
(0)\end{array}$ & $\begin{array}{c}10 / 0 / 0 \\
(0)\end{array}$ & $\begin{array}{c}13 / 0 / 0 \\
(0)\end{array}$ & \begin{tabular}{|c|}
$5 / 0 / 0$ \\
$(0)$
\end{tabular} & \begin{tabular}{|c|}
$3 / 0 / 0$ \\
$(0)$
\end{tabular} & $\begin{array}{c}42 / 0 / 0 \\
(0)\end{array}$ & $\begin{array}{c}10 / 0 / 0 \\
(0)\end{array}$ & - & - & - & $\begin{array}{c}18 / 0 / 0 \\
(0)\end{array}$ & $\begin{array}{c}10 / 2 / 0 \\
(20)\end{array}$ & $\begin{array}{c}19 / 2 / 0 \\
(11)\end{array}$ \\
\hline \begin{tabular}{|l|} 
The Siberian FD \\
(266 samples)
\end{tabular} & \begin{tabular}{|c|}
$2 / 0 / 0$ \\
$(0)$
\end{tabular} & $\begin{array}{c}48 / 0 / 0 \\
(0)\end{array}$ & $\begin{array}{c}55 / 0 / 0 \\
(0)\end{array}$ & $\begin{array}{c}19 / 0 / 0 \\
(0)\end{array}$ & $\begin{array}{c}33 / 0 / 0 \\
(0)\end{array}$ & $\begin{array}{c}25 / 0 / 0 \\
(0)\end{array}$ & $\begin{array}{c}9 / 0 / 0 \\
(0)\end{array}$ & - & $\begin{array}{c}4 / 0 / 0 \\
(0)\end{array}$ & \begin{tabular}{|c|}
$3 / 0 / 0$ \\
$(0)$
\end{tabular} & \begin{tabular}{|c|}
$8 / 0 / 0$ \\
$(0)$
\end{tabular} & \begin{tabular}{|c|}
$10 / 0 / 0$ \\
$(0)$
\end{tabular} & \begin{tabular}{|c|}
$19 / 1 / 0$ \\
$(5)$
\end{tabular} & - & - & $\begin{array}{c}/ 0 / 0 \\
(0)\end{array}$ & - & $\begin{array}{c}5 / 0 / 0 \\
(0)\end{array}$ & \begin{tabular}{|c|}
$12 / 1 / 0$ \\
$(8)$
\end{tabular} & $\begin{array}{c}11 / 2 / 0 \\
(18)\end{array}$ \\
\hline \begin{tabular}{|l|} 
The Ural FD \\
(69 samples)
\end{tabular} & - & \begin{tabular}{|c|}
$5 / 0 / 0$ \\
$(0)$
\end{tabular} & - & \begin{tabular}{|c|}
$12 / 0 / 0$ \\
$(0)$
\end{tabular} & \begin{tabular}{|c|}
$5 / 0 / 0$ \\
$(0)$
\end{tabular} & $\begin{array}{c}10 / 0 / 0 \\
(0)\end{array}$ & - & - & - & - & - & - & \begin{tabular}{|c|}
$5 / 0 / 0$ \\
$(0)$
\end{tabular} & - & - & - & - & \begin{tabular}{|c|}
$21 / 2 / 0$ \\
$(10)$
\end{tabular} & $\begin{array}{c}3 / 0 / 0 \\
(0)\end{array}$ & \begin{tabular}{|c}
$8 / 0 / 0$ \\
$(0)$
\end{tabular} \\
\hline $\begin{array}{l}\text { The North- } \\
\text { western FD } \\
\text { (35 samples) }\end{array}$ & - & $\begin{array}{c}16 / 0 / 0 \\
(0)\end{array}$ & - & $\begin{array}{c}1 / 0 / 0 \\
(0)\end{array}$ & $\begin{array}{c}1 / 0 / 0 \\
(0)\end{array}$ & \begin{tabular}{|c|}
$7 / 0 / 0$ \\
$(0)$
\end{tabular} & - & - & - & - & $\begin{array}{c}1 / 0 / 0 \\
(0)\end{array}$ & - & $\begin{array}{c}1 / 0 / 0 \\
(0)\end{array}$ & - & - & - & - & $\begin{array}{c}5 / 3 / 0 \\
(60)\end{array}$ & $\begin{array}{c}3 / 2 / 0 \\
(67)\end{array}$ & - \\
\hline
\end{tabular}

$\mathrm{N}$ o t e : * means a number of examined samples, items / a number of samples contaminated with DON, items / a number of contaminated samples with DON contents being higher than MPL, items, (frequency of detection, \%). ** is a number of samples received from a given region over the monitoring period from 1999 to 2018. 
Grain samples (271 overall) from Rostov region were provided for monitoring purposes practically every year. DON was detected in grain from 9 out of 17 harvests gathered in different years in concentration varying from 0.05 to $0.77 \mathrm{mg} / \mathrm{kg}$ and frequency of its detection was lower than in Krasnodar region and Adygei Republic. DON contents were higher than MPL only in one out of 271 analyzed samples; it was a grain sample from the harvest gathered in 2000 .

Grain samples from harvests gathered in 1999-2018 in the North Caucasian FD were mostly received from Stavropol region; samples from Kabardino-Balkaria, Dagestan, and North Ossetia-Alania were rarer (harvests gathered in 9 different years). In total 333 grain samples were received to be analyzed over the examined period; 215 out of them were from Stavropol region; 56 samples, from Kabardino-Balkaria and North Ossetia-Alania (Table 2). Frequency of DON detection in contaminated grain harvested in 1999-2018 in Stavropol region varied from $6 \%$ (2009) and $60 \%$ (2017) and maximum contents reached $1.56,0.97$ and $0.73 \mathrm{mg} / \mathrm{kg}$ in some grain samples in 2014, 2017 and 2018 crops accordingly. 9-year monitoring over wheat grain contamination in Kabardino-Balkaria and North Ossetia-Alania revealed the toxin contents in 7 out 9 examined grain harvests (Table 2). DON contents higher than MPL were detected in grains from harvests gathered in 2007, 2010 and 2014 and contamination levels reached $0.91,0.71$ and $0.83 \mathrm{mg} / \mathrm{kg}$ accordingly.

DON content was analyzed in 56 samples of wheat from harvests of 14 different years in the Far Eastern FD. The toxin was detected in wheat from 9 out of 14 grain harvests gathered in Primorye and its contents exceeded MPL in some grain samples in 2008, 2010, 2016 and 2017 crops (Table 2). DON was detected in grains from 4 out of 7 harvests gathered in different years and its contamination levels were higher than MPL in grains in 2016 and 2017 crops.

DON was rarely detected in wheat from the Central FD. The toxin was detected in small quantities, from 0.05 to $0.16 \mathrm{mg} / \mathrm{kg}$, in
$1.4 \%$ out of 693 examined samples of grains from harvests gathered in 20 different years.

Low $(1.2 \%)$ frequency of DON detection was revealed for grain samples received from the Volga FD. The toxin was detected in quantities from 0.09 to $0.37 \mathrm{mg} / \mathrm{kg}$ in few samples out of 331 examined ones. It should be noted that samples contaminated with DON were from harvests gathered in 2017 and 2018.

Low (from 0.18 to $0.25 \mathrm{mg} / \mathrm{kg}$ ) DON contents were detected in very few samples of grain from harvests gathered in 2011, 2017 and 2018 in the Siberian FD.

DON contamination in grain received from the Ural and the Northwestern FDs was analyzed in few grain samples, 69 and 35 accordingly, from harvests gathered in 8 different years. Frequency of the toxin detection was 3 and $14 \%$ accordingly. Contaminated samples were detected only in 2016 and 2017 crops. It should be noted that approximately $2 / 3$ of samples received from the Northwestern FD, namely Kaliningrad region, were contaminated with DON in quantities varying from 0.14 to $0.46 \mathrm{mg} / \mathrm{kg}$. High frequency of DON detection in Kaliningrad region is well in line with data collected in neighboring regions. Thus, DON was detected in $83 \%$ out of 92 examined wheat samples received from Poland and harvested in 2016 in the amount from 0.01 to $1.27 \mathrm{mg} / \mathrm{kg}^{2}$.

Therefore, analysis of dynamics of wheat grain contamination harvested in 1999-2018 in various RF regions revealed a growing trend for DON occurrence not only in the North Caucasian, the Southern, and the Far Eastern FDs where contamination was the highest during the survey but also in the North Western, the Siberian, and the Volga FDs recent years.

Analysis of environmental factors affecting the grain contamination with DON. Climatic and weather conditions are known to have their influence on DON accumulation by toxicogenic fungi of Fusarium genus [14, 15]. We estimated a correlation between grain contamination with DON (Figure 2) and several environmental factors on the example of grain samples from harvests gathered in 2016-2018 in Krasnodar region. The examined factors in- 
cluded the following: average monthly humidity and temperature, a number of sunny hours (a period of time in each month when the sun was above the horizon in a given area and not hidden behind the clouds) and a number of days with precipitations exceeding $1 \mathrm{~mm}$ from May to August. Meteorological data were taken from "Aisori - remote access to DDLarchives", an online achieve that contained data on climatic research ${ }^{7}$.

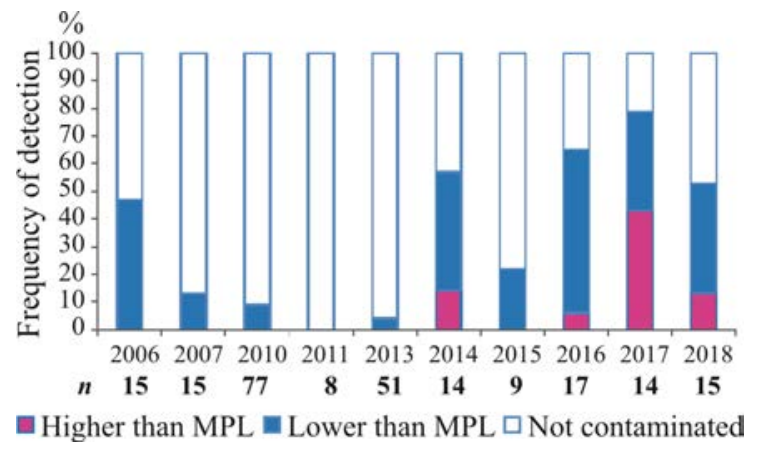

Figure 2. Dynamics of DON contamination in wheat harvested in 2006-2018 in Krasnodar region ( $n$ is a number of samples)

Correlation analysis confirmed there was an authentically significant correlation between frequency of the toxin detection and its average contents in contaminated grain samples harvested in 2006-2018: correlation coefficient was $r=0.68$ and authenticity was $p=0.02$ at the significance level $\alpha=0.05$. Keeping in mind that variation coefficient for frequency of DON detection was significantly lower we took the former coefficient to use in further statistical analysis as a leading one. We didn't detect any correlation between frequency of DON detection and the examined weather factors from June to August. At the same time weather conditions in May, namely duration of sunny weather and a number of days with precipitations exceeding $1 \mathrm{~mm}$ authentically had the most significant influence on DON contamination, the significance level being 0.05 (Table 3). These data are well in line with the fact that wheat grain is the most susceptible to fungi infections during its milky ripeness and in Krasnodar region this period is from mid May to mid June. Clear and sunny weather and a decrease in a number of rainy days in May make for declining wheat contamination with DON and vice versa.

The detected correlation was confirmed with using Mann - Whitney non-parametric test. To apply it, we divided a parameter within a month into two class-intervals, upper and lower ones, and frequencies of DON detection were written down in a relevant classinterval. The analysis revealed that authenticity was higher than significance $(p>0.05)$ for average monthly air humidity and temperature in May; hence, they had only insignificant influence on contamination levels. But the significance was $p<0.05$ for duration of sunny days and a number of rainy days with precipitation exceeding $1 \mathrm{~mm}$ and this indicates there is an authentic correlation between these two weather factors and grain contamination.

Table 3

Correlations between frequency of DON detection in wheat grain and examined weather conditions for harvests gathered in 2006-2018

\begin{tabular}{|l|c|c|c|c|}
\hline \multicolumn{1}{|c|}{ Parameter } & \multicolumn{3}{|c|}{ Correlation coefficient, $r$ (authenticity, $p$ ) } \\
\hline Month & May & June & July & August \\
\hline Average monthly air humidity & $0.49(0.120)$ & $-0.05(0.868)$ & $-0.22(0.504)$ & $-0.08(0.811)$ \\
\hline Average monthly air temperature & $-0.46(0.150)$ & $-0.03(0.916)$ & $0.02(0.946)$ & $0.12(0.718)$ \\
\hline Duration of sunny hours per month & $-\mathbf{0 . 6 5 ( 0 . 0 2 9 )}$ & $-0.08(0.805)$ & $-0.07(0.837)$ & $-0.09(0.782)$ \\
\hline $\begin{array}{l}\text { A number of days with precipitations } \\
\text { exceeding 1 mm }\end{array}$ & $\mathbf{0 . 7 4 ( \mathbf { 0 . 0 0 8 } )}$ & $-0.21(0.525)$ & $-0.11(0.747)$ & $-0.11(0.756)$ \\
\hline
\end{tabular}

\footnotetext{
${ }^{7}$ Veselov V.M., Pribyl'skaya I.R., Mirzebasov O.A. Spetsializirovannye massivy dlya klimaticheskikh issledovanii. «Aisori Udalennyi dostup k YaOD-arkhivam [Specialized data arrays for climatic research. "Aisori - remote access to DDL-archieves"]. VNIIGMI-MC Aisori D. Available at: http://aisori-m.meteo.ru/waisori/index0.xhtml (March 03, 2021) (in Russian).
} 
Table 4

Two-factor dispersion analysis of a grain quality category, year of a harvest, and contamination

\begin{tabular}{|l|c|c|c|c|c|}
\hline \multicolumn{7}{|c|}{ Dependent variable (DON content in a sample) } \\
\hline \multicolumn{1}{|c|}{ Source } & Sum of type III squares & Degree of freedom & Mean square & F-criterion & Significance \\
\hline Adjusted model & $3.422^{\mathrm{a}}$ & 3 & 1.141 & 1.300 & 0.284 \\
\hline Year & 0.503 & 1 & 0.503 & 0.573 & 0.452 \\
\hline Grain quality category & 0.811 & 1 & 0.811 & 0.924 & 0.341 \\
\hline Year $\times$ category & 2.034 & 1 & 2.034 & 2.319 & 0.134 \\
\hline Error & 47.380 & 54 & 0.877 & & \\
\hline Total & 68.325 & 58 & & & \\
\hline Adjusted result & 50.802 & 57 & & & \\
\hline a - R-square $=0.067$ (Adjusted R-square $=0.016)$ & \multicolumn{5}{l}{} \\
\hline
\end{tabular}

Table 5

Frequency and levels of DON contamination in food barley, oats, rye, and corn harvested in 1989-2018

\begin{tabular}{|c|c|c|c|c|c|c|c|}
\hline \multirow{3}{*}{ Grain } & \multicolumn{3}{|c|}{ Number of samples } & \multirow{3}{*}{$\begin{array}{c}\text { DON contents in } \\
\text { contaminated sam- } \\
\text { ples, } \mathrm{mg} / \mathrm{kg}\end{array}$} & \multicolumn{3}{|c|}{$\begin{array}{c}\text { DON contents in total } \\
\text { samples, } \mathrm{mg} / \mathrm{kg}\end{array}$} \\
\hline & \multirow{2}{*}{ Total, item } & Containing DON & DON contents higher than MPL & & $M$ & $M e$ & $90 \%$ \\
\hline & & \multicolumn{2}{|c|}{ items ( $\%$ of total number) } & & $M$ & Me & $90 \%$ \\
\hline Barley & 1,293 & $54(4.2)$ & $5(0.4)$ & $0.05-8.95$ & 0.02 & 0 & 0 \\
\hline Corn & 278 & $33(11.9)$ & MPL not fixed & $0.05-0.95$ & 0.04 & 0 & 0.07 \\
\hline Oats & 200 & $6(3.0)$ & MPL not fixed & $0.06-0.96$ & 0.01 & 0 & 0 \\
\hline Rye & 1,020 & $6(0.6)$ & MPL not fixed & $0.06-0.44$ & 0.00 & 0 & 0 \\
\hline
\end{tabular}

N o t e : DON MPL, mg/kg, not exceeding 1.0 for barley (TR CU 015/2011 “Safety of grain”).

There was a growth in grain contamination observed from 2006 to 2018. Correlation analysis revealed a directly proportional correlation between a year of a harvest and frequency of DON detection: correlation coefficient was $r=0.60$ and authenticity was $p=0.049$ at the significance level $\alpha=0.05$. Although it seems hardly possible to use this correlation to predict future situation due to $\alpha \approx p$, we still can't ignore it.

We performed two-factor dispersion analysis of a grain quality category, a year of a harvest, and DON contents in a sample (Table 4). A year of a harvest was used as fixed factor A, and grain quality category, fixed factor B.

Analysis of barley, oats, rye and corn contamination harvested in 1989-2018. Aggregated data on DON contamination in barley, oats, rye, and corn harvested in 1989-2018 are given in Table 5. DON contamination in food barley was examined on the example 1,293 grain samples harvested in 1989-2018. Frequency of DON contamination in barley was lower than in wheat $(10 \%)$ and corn. DON was detected in $4 \%$ samples (54 overall) in quantities varying from 0.05 to $8.95 \mathrm{mg} / \mathrm{kg}$, and 5 samples contained DON in quantities higher than MPL. DON was detected most frequently in grains in 2017 and 2014 crops, 44 and $30 \%$ accordingly, and this frequency was substantially lower in grains harvested in $2015(22 \%)$, 2016 (19\%), 1989 (16\%), 1992 and 2009 (14\% in both these years). In other years, frequency varied from 0 to $7 \%$. Notably, over the recent years, starting from 2014, DON contamination in barley has been growing.

Approximately $80 \%$ of all barley production is located in the Central, the Volga, the Southern and the Siberian FDs [24]. The greatest number of samples was received from the Central and the Southern FDs. The toxin was detected in $11 \%$ samples from the Southern FD in quantities varying from 0.05 to $1.7 \mathrm{mg} / \mathrm{kg}$ and its contents were higher than MPL in one sample of grain harvested in 2017. Barley received from Krasnodar region was the most contaminated with DON was, since $23 \%$ out of 39 samples were contaminated 
with DON in the amount from 0.05 to $1.7 \mathrm{mg} / \mathrm{kg}$; the least contaminated barley was received from Rostov region: $7.5 \%$ out of 40 samples were contaminated with DON in the amount from 0.05 to $0.11 \mathrm{mg} / \mathrm{kg}$. According to our data the toxin was most frequently detected in barley received from the North Caucasian FD; DON was detected in $17 \%$ of 64 examined samples, in particular, in low quantities in samples from Stavropol region $(0.10$ and $0.11 \mathrm{mg} / \mathrm{kg}$ ). The range of DON contamination in grain from Kabardino-Balkaria was wider, from 0.07 to $8.95 \mathrm{mg} / \mathrm{kg}$ with exceeding MPL DON in grain harvested in 2017. DON in low quantities was detected in rare cases in grain received from Ingushetia and North Ossetia.

Although samples from the Far Eastern FD were sent for analysis irregularly and in relatively few numbers, approximately half of examined samples (10 out of 21) were contaminated with DON in quantities varying from 0.05 to $2.83 \mathrm{mg} / \mathrm{kg}$ with exceeding of MPL in one sample harvested in Amur region in 2018. Barley lots harvested in 2005-2009 in Primorye contained the toxin in low quantities, from 0.06 to $0.21 \mathrm{mg} / \mathrm{kg}$.

Our research results revealed that, just as it was the case with wheat, samples received from the Far Eastern, the North Caucasian, and the Southern FDs were the most contaminated.

Corn grain was similar to wheat grain $(11.98 \%$ against $10 \%)$ as per DON contaminations. DON contents in contaminated corn samples varied from 0.05 to $0.95 \mathrm{mg} / \mathrm{kg}$ (average contents amounted to $0.04 \mathrm{mg} / \mathrm{kg}$ ). For reference, DON contents in contaminated wheat samples amounted to $0.05-6.65 \mathrm{mg} / \mathrm{kg}$. Having analyzed dynamics of domestic corn contamination with DON, we revealed that DON occurred only in grains harvested in 2000, 2002, and 2012-2018, and the highest frequency of DON contamination was detected in recent years $(2014,2016-2018)$.

The Southern, the North Caucasian and the Central FDs are basic corn producers in Russia $^{8}$. The greatest number of samples was sent for analysis from the Southern (217) and the North Caucasian FDs (47). The toxin was the most frequently detected in corn from the North Caucasian FD, namely, in $32 \%$ out of 47 examined lots, in quantities varying from 0.05 to $0.68 \mathrm{mg} / \mathrm{kg}, 0.09 \mathrm{mg} / \mathrm{kg}$ on average. The toxin was detected less frequently, namely, in $6 \%$ out of samples received from the Southern FD; its amount varied from 0.05 to $0.95 \mathrm{mg} / \mathrm{kg}, 0.02 \mathrm{mg} / \mathrm{kg}$ on average. DON was detected in rare corn lots received from the Central FD in quantities equal to 0.29 and $0.32 \mathrm{mg} / \mathrm{kg}$. It should be noted that 4 out of 6 examined corn samples received from the Far Eastern FD was contaminated with DON in quantities varying from 0.13 to $0.55 \mathrm{mg} / \mathrm{kg}$.

Oats contamination with DON was examined in 200 samples of grains harvested in 1999-2018. The toxin was detected in $3 \%$ cases in quantities from 0.05 to $0.96 \mathrm{mg} / \mathrm{kg}$, $0.008 \mathrm{mg} / \mathrm{kg}$ on average (Table 5). DON was detected in grains harvested in 2009, 2016 and 2017.

It should be noted that approximately $39 \%$ of overall oats produced in the country is grown in Altai, Krasnoyarsk, Novosibirsk, Tyumen, Kemerovo, and Omsk regions and Bashkortostan [25]. The toxin was not detected in samples received from these regions. Contamination was detected only in samples from the Far Eastern (4 cases) and the North Caucasian FD (2 cases). Oats samples from Amur region and Primorye were the most contaminated with DON, its amounts varying from 0.09 to $0.96 \mathrm{mg} / \mathrm{kg}$. The toxin was less frequently detected and in lower quantities in oats received from Ingushetia and Stavropol region $(0.06 ; 0.09 \mathrm{mg} / \mathrm{kg}$ accordingly).

Generally the toxin was detected in rye samples were received from the North Caucasian FD during epiphytoties (harvests gathered in 1989 and 1992). The toxin was detected in rare cases in low quantities in grains harvested in 1996 and 2007, at the level of 0.22 and 0.06 $\mathrm{mg} / \mathrm{kg}$ in the North Caucasian and the Central FDs accordingly.

\footnotetext{
${ }^{8}$ Sel'skoe khozyaistvo v Rossii. 2019: statisticheskii sbornik [Agriculture in Russia. 2019: statistical data collection]. Rosstat. Moscow, 2019, 91 p. (in Russian).
} 
Approximately $74.4 \%$ of the total grain harvest of rye in the country is grown in the Volga FD, Bashkortostan, Tatarstan, Orenburg and Saratov regions [24]. Rye samples received from these regions were not contaminated with DON.

According to monitoring results, it can be concluded that wheat and corn are the most frequently contaminated with DON among grain, and, barley to a lesser extent. The growth in DON occurrence in grains in recent years (2014, 2017 and 2018) could be explained not only by weather conditions auspicious for toxin production by Fusarium genus but also by changes in F. graminearum areas. According to Gagkaeva and others (2014), over the last few years these fungi spread into new territories located to the north from their usual habitat [13]. Starting from 2003, resistant populations of $F$. graminearum species occurred in Primorye and North Caucasus, spread into new areas, for example, into the north-western part of Russia, during local weather changes characterized with climatic conditions auspicious for microfungi [24]. The authors believe that the existing climate warming, especially in winter, makes for F. graminearum survival in new areas and the fungi get adapted to colder environment [13]. Agriculture intensification might be another reason for growing DON contamination.

Monitoring results confirmed that the Southern, the North Caucasian, and Far Eastern FDs remained the primary areas where Fusarium head blight was widely spread. At the same time in 2016-2018 frequency of DON detection grew in areas located to the north from the aforementioned regions.

Assessment of health risks caused by DON contaminations in food wheat grains. Wheat is a primary source of DON intake with food. When calculating probable DON intake into the human body, we used average mycotoxin contents in samples of grains harvested in the same year $(M$, Table 1$)$. Toxin intake per person was calculated within a sce- nario when DON contents in food products made from processed wheat corresponded to its contents in grain; that is, processing didn't produce any significant effects on DON contents in a finished product. This approach corresponds to results obtained in studies on stability of the toxin during food processing and cooking: when grain is refined, the toxin contents usually remain the same or go down to 22-23\% [15, 27]; any thermal treatment doesn't influence DON contents [15, 27, 28].

Data on consumption of wheat products (wheat bread, grocery, flour confections, wheat flour, semolina, and macaroni) were taken from budget statistical reports issued by the Federal State Statistic Service and based on a sample survey over budgets spent by households ${ }^{9}$. Average consumption of food products made of processed wheat decreased over the last 25 years, from $267 \mathrm{~g}$ in 1993 to $209 \mathrm{~g}$ in 2018; it was taken into account when DON intake was calculated. Average person's weight was taken as equal to $60 \mathrm{~kg}$.

Calculated total DON intake per person on average in Russia was significantly different from year to year. DON intake varied from $0.2 \%$ (in 1999) to $140 \%$ (in 1992) of DON PMTDI but remained lower than fixed DON PMTDI, $1 \mu \mathrm{g} / \mathrm{kg}$ of body weight, in the most cases except in 1992 (140\%) and 2014 (102\%) (Table 6) [20].

We should note that high average calculated DON intake with food products made of wheat harvested in 2014 for the country population didn't differ significantly from the intake calculated for the most risky regions; it was partly due to the fact that $88 \%$ of all grain samples for analysis were received from the Southern and the North Caucasian FDs. More profound analysis of estimated daily DON intakes revealed that toxin intake was higher in the Southern and the North Caucasian FDs than on average in the country during the survey and varied from $3 \%$ (in 2004 and 2009) to $410 \%$ of PMTDI (in 1992). Calculated DON intake was also higher than PMTDI in the

\footnotetext{
${ }^{9}$ Potreblenie produktov pitaniya v domashnikh khozyaistvakh (byulleten') [Food products consumption in households (bulletin)]. Rosstat. Available at: https://rosstat.gov.ru/compendium/document/13292 (March 03, 2021) (in Russian).
} 
Table 6

Calculated daily DON intake with food products made of wheat grain harvested in 1989-2018

\begin{tabular}{|c|c|c|}
\hline \multirow{2}{*}{ A harvest year } & \multicolumn{2}{|c|}{ Estimated daily intake $\left(N_{\text {calc. }}\right), \mu \mathrm{g} / \mathrm{kg}$ of b.w. a day ( $\%$ of PMTDI) } \\
\hline & On average in Russia & The Southern and the North Caucasian FD \\
\hline 1989 & $0.96(96.0)$ & - \\
\hline $1990-1991$ & $0.07(7.0)$ & - \\
\hline 1992 & $1.40(140.0)$ & $4.10(410.0)$ \\
\hline 1993 & $0.89(89.0)$ & $2.18(218.0)$ \\
\hline 1994 & $0.12(12.0)$ & $0.29(29.0)$ \\
\hline 1995 & $0.12(12.0)$ & $0.68(68.0)$ \\
\hline 1996 & $0.07(7.0)$ & $0.18(18.0)$ \\
\hline 1997 & $0.08(8.0)$ & $0.20(20.0)$ \\
\hline 1998 & $0.12(12.0)$ & $0.48(48.0)$ \\
\hline 1999 & $0.002(0.2)$ & - \\
\hline 2000 & $0.04(0.4)$ & $0.30(30.0)$ \\
\hline 2001 & $0.036(3.6)$ & $0.22(22.0)$ \\
\hline 2002 & $0.028(2.8)$ & $0.28(28.0)$ \\
\hline 2003 & $0(0)$ & $0(0)$ \\
\hline 2004 & $0.004(0.4)$ & $0.03(3.0)$ \\
\hline 2005 & $0.084(8.4)$ & $0.36(36.0)$ \\
\hline 2006 & $0.096(9.6)$ & $0.18(18.0)$ \\
\hline 2007 & $0.066(6.6)$ & $0.24(24.0)$ \\
\hline 2008 & $0.07(7.0)$ & $0.17(17.0)$ \\
\hline 2009 & $0.025(2.5)$ & $0.03(3.0)$ \\
\hline 2010 & $0.18(18.0)$ & $0.14(14.0)$ \\
\hline 2011 & $0.04(4.0)$ & $0.12(12.0)$ \\
\hline 2012 & $0(0)$ & $0(0)$ \\
\hline 2013 & $0.04(4.0)$ & $0.04(4.0)$ \\
\hline 2014 & $1.02(102.0)$ & $1.12(112.0)$ \\
\hline 2015 & $0.05(5.0)$ & $0.05(5.0)$ \\
\hline 2016 & $0.16(16.0)$ & $0.26(26.0)$ \\
\hline 2017 & $0.63(63.0)$ & $1.09(109)$ \\
\hline 2018 & $0.12(12.0)$ & $0.56(56.0)$ \\
\hline
\end{tabular}

Southern and the North Caucasian FDs in 1993, 2014, and 2017 and accounted for 218, 112 and $109 \%$ of PMTDI accordingly. The aforementioned ascending trend in wheat contamination calls for implementing necessary activities aimed at control over food grain safety.

Conclusions. We have analyzed the results obtained through long-term monitoring over food grain contamination with mycotoxins accomplished by the Federal Service for Surveillance over Consumer Rights Protection and Human Well-being with active participation by the Federal Research Center of Nutrition, Biotechnology, and Food Safety. The analysis confirmed that grains grown in the Southern, the North Caucasian, and the Far Eastern FDs were the most susceptible to DON contamination. At the same time, there is a growth in frequency of DON detection in grains grown in more northern regions and this indicates that Fusarium head blight is spreading over new areas and grain contamination with mycotoxins producers is becoming more probable. This area of contamination can increase when grain is transported from one region to another and this means it is necessary to control grain quality, primarily, when it is used as seed grain.

The highest frequency of DON detection and highest DON contents are typical for wheat and corn among all examined grains. It was shown that calculated daily DON intake with food wheat products was higher than PMTDI DON set by JECFA in some years over the examined period. DON intake was more likely to exceed PMTDI for people living in the Southern and the North Caucasian FDs. 
To reduce health risks caused by food grain contamination with DON, it is vital to implement activities aimed at stricter control over spread of Fusairum head blight in the RF. Special attention should be paid to monitoring over safety of wheat and corn food grains, in particular, those grown in the Southern and the North Caucasian FDs.
Funding. The research was accomplished within the Research work No. 0529-2019-0057 "Development of a system for quality and safety of food products including food additives and alcoholic beverages produced with biotechnologies".

Conflict of interests. The authors declare there is no any conflict of interests.

\section{References}

1. Bryden W.L. Mycotoxins in the food chain: human health implications. Asia Pac. J. Clin. Nutr., 2007, vol. 16, pp. 95-101.

2. Tutel'yan V.A., Kravchenko L.V., Sergeev A.Yu. Mycotoxins. Mycology today. In: Yu.T. D'yakova, Yu.V. Sergeeva eds., Moscow, Natsional'naya akademiya mikologii Publ., 2007, vol. 1, pp. 283-304 (in Russian).

3. Birr T., Hasler M., Verreet J.-A., Klink H. Composition and Predominance of Fusarium Species Causing Fusarium Head Blight in Winter Wheat Grain Depending on Cultivar Susceptibility and Meteorological Factors. Journal of Plant Diseases and Protection, 2020, vol. 8, no. 4, pp. 617. DOI: 10.3390/microorganisms8040617

4. Bryla M., Waskiewicz A., Podolska G., Szymczyk K., Jedrzejczak R., Damaziak K., Sulek A. Occurrence of 26 Mycotoxins in the Grain of Cereals Cultivated in Poland. Toxins, 2016, vol. 8, no. 6, pp. 160. DOI: $10.3390 /$ toxins 8060160

5. Fredlund E., Gidlund A., Sulyok M., Börjesson T., Krska R., Olsen M., Lindblad M. Deoxynivalenol and other selected Fusarium toxins in Swedish oats - Occurrence and correlation to specific Fusarium species. Int. J. Food. Microbiol., 2013, vol. 167, pp. 276-283.

6. Xu W., Han X., Li F. Co-occurrence of multi-mycotoxins in wheat grains harvested in Anhui province, China. Food Control, 2019, vol. 96, pp. 180-185. DOI: 10.1016/j.foodcont.2018.09.006

7. Cheli F., Pinotti L., Novacco M., Ottoboni M., Tretola M., Dell'Orto V. Mycotoxins in Wheat and Mitigation Measures. IntechOpen, 2017. Available at: https://www.intechopen.com/chapters/53908 (17.01.2021).

8. Bertuzzi T., Leggieri M.C., Battilani P., Pietri A. Co-occurrence of type A and B trichothecenes and zearalenone in wheat grown in northern Italy over the years 2009-2011. Food Addit. Contam. Part B, 2014, vol. 7, pp. 273-281.

9. Darsanaki R.K., Issazadeh K., Aliabadi M.A., Chakoosari M.M.D. Occurrence of Deoxynivalenol (DON) in wheat flours in Guilan Province, Northern Iran. Ann. Agric. Environ. Med., 2015, vol. 22, pp. 35-37.

10. Alshannaq A., Yu O.-H. Occurrence, Toxicity, and Analysis of Major Mycotoxins in Food. Int. J. Environ. Res. Public Health, 2017, vol. 14, pp. 632. DOI: 10.3390/ijerph14060632

11. Opinion of the Scientific Panel n Contaminants in the Food Chain (CONTAM Panel) on the risks to human and animal health related to the presence of deoxynivalenol and its acetylated and modified forms in food and feed. European Food Safety Authority. EFSA Journal, 2017, vol. 15, no. 9. DOI: $10.2903 /$ j.efsa.2017.4718

12. Khaneghah A.M., Martins L.M., von Hertwig A.M., Bertoldo R., Sant'Ana A.S. Deoxynivalenol and its masked forms: Characteristics, incidence, control and fate during wheat and wheat based products processing - A review. Trends in Food Science \& Technology, 2018, vol. 71, pp. 13-24. DOI: 10.1016/j.tifs.2017.10.012

13. Gagkaeva T.Yu., Gavrilova O.P., Levitin M.M. Biodiversity and distribution of the main toxigenic Fusarium fungi. Biosfera, 2014, vol. 6, no. 1, pp. 36-45 (in Russian).

14. Codex Alimentarius. CXC 51-2003 Code of Practice for the Prevention and Reduction of Mycotoxin Contamination in Cereal. WHO Food Standards Programme, Food and Agriculture Organization of the United Nations. Available at: http://www.fao.org/fao-who-codexalimentarius/sh-proxy/en/?lnk= $1 \&$ url=https $\% 253 \mathrm{~A} \% 252 \mathrm{~F} \% 252 \mathrm{Fworkspace}$.fao.org $\% 252 \mathrm{Fsites} \% 252 \mathrm{Fcodex} \% 252 \mathrm{FStandards} \% 252 \mathrm{FCX}$ C\%2B51-2003\%252FCXC_051e.pdf(29.01.2021). 
15. Machikhina L.I., Alekseeva L.V., L'vova L.S. Nauchnye osnovy prodovol'stvennoi bezopasnosti zerna (khranenie i pererabotka) [Scientific foundations of food grain safety storage and processing]. Moscow, DeLi print Publ., 2007, 382 p. (in Russian).

16. Bianchini A., Horsley R., Jack M.M., Kobielush B., Ryu D., Tittlemier Sh., Wilson W.W., Abbas H.K. [et al.]. DON Occurrence in Grains: A North American Perspective. Cereal foods world, 2015, vol. 60, no. 1, pp. 32-56. DOI: 10.1094/CFW-60-1-0032

17. Bottalico A., Perrone G. Toxigenic Fusarium species and mycotoxins associated with head blight in small-grain cereals in Europe. European Journal of Plant Pathology, 2002, vol. 108, pp. 611-624.

18. Tutelyan V.A. Deoxynivalenol in cereals in Russia. Toxicol. Lett., 2004, vol. 153, no. 1, pp. 173-179. DOI: 10.1016/j.toxlet.2004.04.042

19. Zhao Yu., Guan X., Zong Yi., Hua X., Xing F., Wang Y., Wang F., Liu Y. Deoxynivalenol in wheat from the Northwestern region in China. Food Additives \& Contaminants: Part B, 2018, vol. 11, no. 4, pp. 281-285. DOI: 10.1080/19393210.2018.1503340

20. JECFA. Summary of toxicological evaluations. Summary Report of the 72nd Meeting of the Joint FAO.WHO Expert Committee on Food Additives, Food and Agriculture Organization of the United Nations, Rome, 2010. Available at: www.who.int/foodsafety/chem/summary72_rev.pdf (04.02.2021).

21. Pestka J.J. Deoxynivalenol: Mechanisms of action, human exposure, and toxicological relevance. Arch. Toxicol., 2010, vol. 84, pp. 663-679.

22. Codex Alimentarius. CXS 193-1995 General Standard for Contaminants and Toxins in Food and Feed (last amended in 2019). WHO Food Standards Programme, Food and Agriculture Organization of the United Nations. Available at: http://www.fao.org/fao-who-codexalimentarius/sh-proxy/en/?lnk= $1 \&$ url=https $\% 253 \mathrm{~A} \% 252 \mathrm{~F} \% 252 \mathrm{Fw}$ orkspace.fao.org $\% 252 \mathrm{Fsites} \% 252 \mathrm{Fcodex} \% 252 \mathrm{FStandards} \% 252 \mathrm{FCX}$ S\%2B193-1995\%252FCXS_193e.pdf(03.03.2021).

23. COMMISSION REGULATION (EC) No $1881 / 2006$ of 19.12 .2006 setting maximum levels for certain contaminants in foodstuffs. Available at: https://eur-lex.europa.eu/LexUriServ/LexUriServ.do?uri= OJ:L:2006:364:0005:0024: EN:PDF (03.03.2021).

24. Bryła M., Ksieniewicz-Wo'zniak E., Wa'skiewicz A., Szymczyk K., J drzejczak R. Natural Occurrence of nivalenol, deoxynivalenol, and deoxynivalenol-3-glucoside in polish winter wheat. Toxins, 2018, vol. 10, no. 2, pp. 81. DOI:10.3390/toxins10020081

25. Gavrilova O.P., Gagkaeva T.Yu. Fuzarioz zerna na severe Nechernozem'ya i v Kaliningradskoi oblasti v 2007-2008 godakh [Fusarium grain blight in the north of the Non-Black Earth Region and in the Kaliningrad Region in 2007-2008]. Zashchita i karantin rastenii, 2010, no. 2, pp. 23-25 (in Russian).

26. Prodovol'stvennaya nezavisimost' Rossii: v 2 t. [Food independence of Russia: in 2 volumes]. In: A.V. Gordeev ed. Moscow., OOO Tekhnologiya TsD Publ., 2016, vol. 1, 560 p. (in Russian).

27. Kushiro M. Effects of Milling and Cooking Processes on the Deoxynivalenol Content in Wheat. Int. J. Mol. Sci., 2008, vol. 9, pp. 2127-2145. DOI: 10.3390/ijms9112127

28. Karlovsky P., Suman M., Berthiller F., De Meester J., Eisenbrand G., Perrin I., Oswald I.P., Speijers G., Chiodini A., Recker T., Dussort P. Impact of food processing and detoxification treatments on mycotoxin contamination. Mycotoxin Res., 2016, vol. 32, pp. 179-205.

Sedova I.B., Zakharova L.P., Kiseleva M.G., Chalyy Z.A., Timonin A.N., Aristarkhova T.V., Kravchenko L.V., Tutelyan V.A. Deoxinivalenol as a risk factor of food grain contamination: monitoring results of 1989-2018 years harvests in Russian Federation. Health Risk Analysis, 2021, no. 3, pp. 84-97. DOI: 10.21668/health.risk/2021.3.08.eng

Received: 31.03.2021

Accepted: 27.07.2021

Published: 30.09 .2021 